\title{
The PLANTS System: Enabling Mixed Societies of Communicating Plants and Artefacts
}

\author{
Christos Goumopoulos, Eleni Christopoulou, Nikos Drossos, and Achilles Kameas \\ Computer Technology Institute, Research Unit 3, Design of Ambient Intelligent Systems \\ Group, Patras, Hellas \\ \{goumop, hristope, ndrossos, kameas\}@eti.gr
}

\begin{abstract}
In this paper we discuss research work that enables the development of mixed societies of communicating plants and artefacts. PLANTS is an EUfunded Research and Development project, which aims to investigate methods of creating "interfaces" between artefacts and plants in order to enable people to form mixed, interacting (potentially co-operating) communities. Amongst others the project aims to develop hardware and software components that should enable a seamless interaction between plants and artefacts in scenarios ranging from domestic plant care to precision agriculture. This paper deals with the approach that we follow for the development of the homonymous system and discusses its architecture with special focus on describing the communication among artefacts and plants and on designing an ontology that provides a formal definition of the domain under consideration.
\end{abstract}

\section{Introduction}

The vision of Ambient Intelligence (AmI) [1] implies that technology will become invisible, embedded in our natural surroundings, present whenever we need it, enabled by simple and effortless interactions, accessed through multimodal interfaces, adaptive to users and context and proactively acting. In the future, it is envisaged that the spaces we live in will be populated by many thousands of everyday objects with the ability to sense and actuate in their environment, to perform localised computation, and to communicate, even collaborate, with each other. These objects are identified as artefacts and are playing a large role in research towards intelligent systems and ubiquitous computing. The AmI environment can be considered to host several Ubiquitous Computing (UbiComp) applications, which make use of the infrastructure provided by the environment and the services provided by the objects therein.

An important characteristic of AmI environments is the merging of physical and digital space; major research efforts are currently targeting the "disappearance" of the computer into the fabric of our environment [2]. However, currently there are only few discussions on including elements of real (natural) environment into UbiComp applications. In this paper, we present our efforts to create digital interfaces to nature, in particular to selected species of plants, enabling the development of synergistic and scalable mixed communities of communicating artefacts and plants. 
Plants are ubiquitous in the sense that they exist almost in every environment populated by humans. They easily adapt to changing conditions and they can be used as sources of complex information on the environment. Our approach builds upon previous experience gained in the e-Gadgets project [3], where ubiquitous computing applications are composed from everyday objects under the Gadgetware Architectural Style (GAS) [4]. We have extended this concept to regard plants as real-world "components", which can communicate with artefacts in the digital space by using compatible metaphors. To this end, we provide each plant with a GAS-compatible description of its properties and state.

In order to create synergistic mixed communities of communicating artefacts and plants, an interdisciplinary research effort is required involving plant science domain knowledge, sensory hardware engineering and ubiquitous system software engineering [5]. Research issues include the selection of the "right" sensors with respect to the data and sensitivity, the fusion of sensed data and its semantic characterization, the synchronization of the entire artefact-plants system, the integration of sensors, actuators, artefacts with decision making procedures etc.

The rest of the paper is organized as follows. Section 2 outlines an overview of the PLANTS system where basic concepts and components are identified and described. In section 3 the architecture of the system and design decisions are discussed. The role and the design of the PLANTS ontology are presented in section 4 . Section 5 discusses scenarios that have been examined for showcasing the developments. Section 6 examines related work and emphasizes on the different perspectives of our work. Finally, section 7 concludes this paper.

\section{Overview of the PLANTS System}

We are motivated from the fact that plants are truly ubiquitous entities, since they exist in everyday environments. In addition, plants can be used either as "biosensors" or as a "natural" and beautiful interface to services. In the former case, the context provided by digital sensors or artefacts can be enhanced with data of biological nature, which, if fused together, can trigger a more contextually accurate response; in the latter case, artefacts can use plants as a front end to delivering specific services (i.e. greetings, narration, alarms, etc). Thus, plants, if turned to ePlants, can become part of a range of UbiComp applications ranging from agricultural to domestic.

A mechanism for low-level context acquisition, which reads plant signals from sensors is the first step in the plant context management process (Fig. 1). This information is probably not in a format that can be used by a system in order to make decisions or reach a conclusion. In a second step the plant signals are interpreted and a high-level context information is derived. For example, a sensor that measures the temperature of a plant may use a metric system that is different than the Celsius system. In that case the interpretation simply maps the sensor readings to the Celsius metric system and the information can be displayed to a device if we choose so. Aggregation of context is also possible meaning that semantically richer information may be derived based on the fusion of several measurements that come from different homogeneous or heterogeneous sensors located on plants or artefacts. To determine 
photo-oxidative stress, for example, requires monitoring of chlorophyll florescence and ambient light level signals to adjust supplementary light. As another example, determining water stress requires monitoring of plant's leaf temperature, ambient temperature and humidity level [5].

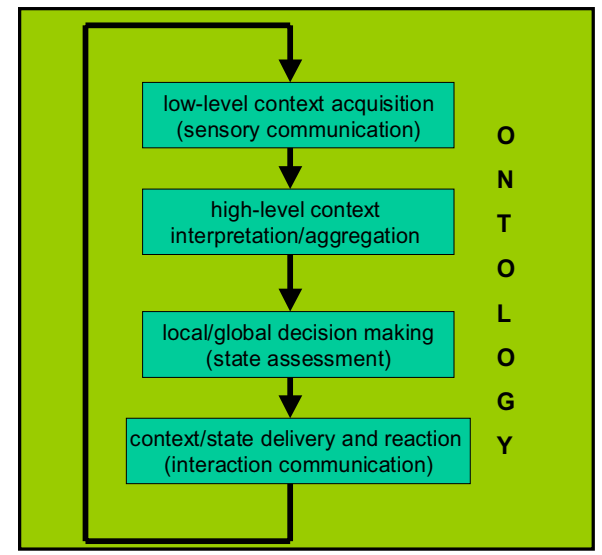

Fig. 1. Plant/environmental context management process

Having acquired the necessary context we are in a position to assess a state of the plant and decide appropriate response activation. Adopting the definition from Artificial Intelligence, a state is a logical proposition defined over a set of context measurements [6]. This state assessment will be based on a set of rules that Plant Scientists have researched and which the PLANTS system has to encode in a flexible and extensible manner. The low (sensor) and high (fused) level data, their interpretation and the decision-making rules are encoded in an ontology.

The reaction may be as simple as turn on a light or send a message to the user or a composite one such as request watering to the pot in case of drought stress or as spraying mist in case of heat stress, which means that the system has to differentiate between the two kinds of water stress. Such a decision may be based on local context or may require context from external sources as well, e.g., weather station supporting prediction of plant disease spreading.

\section{System Components}

A mixed society of plants and artefacts can be regarded as a distributed system, which will globally manage the resources of the society, its function(s) and its interaction with the environment. The PLANTS system is schematically represented in Fig 2; the UbiComp applications, which use mixed societies of 'ePlants' and 'eGadgets', are referred to as 'bioGadgetworlds' and are composed of a number of basic components:

ePlants: Plants are transformed into 'ePlants' through the superimposition of a technological layer and may represent either a specific plant or a set of plants (a set may be defined in terms of specific plant species or a number of plants in a particular location). The scope of the system enables groups of ePlants to be organized into a 
large number of nodes, to create a hierarchical structure that evenly distributes the communication load and other resource (power, memory, computation) consumption and also facilitates distributed decision-making. These nodal groupings are considered as 'high-level' ePlants with the hierarchical structure adhering to the philosophy of the software component-engineering paradigm where composite components are synthesized from simpler ones.

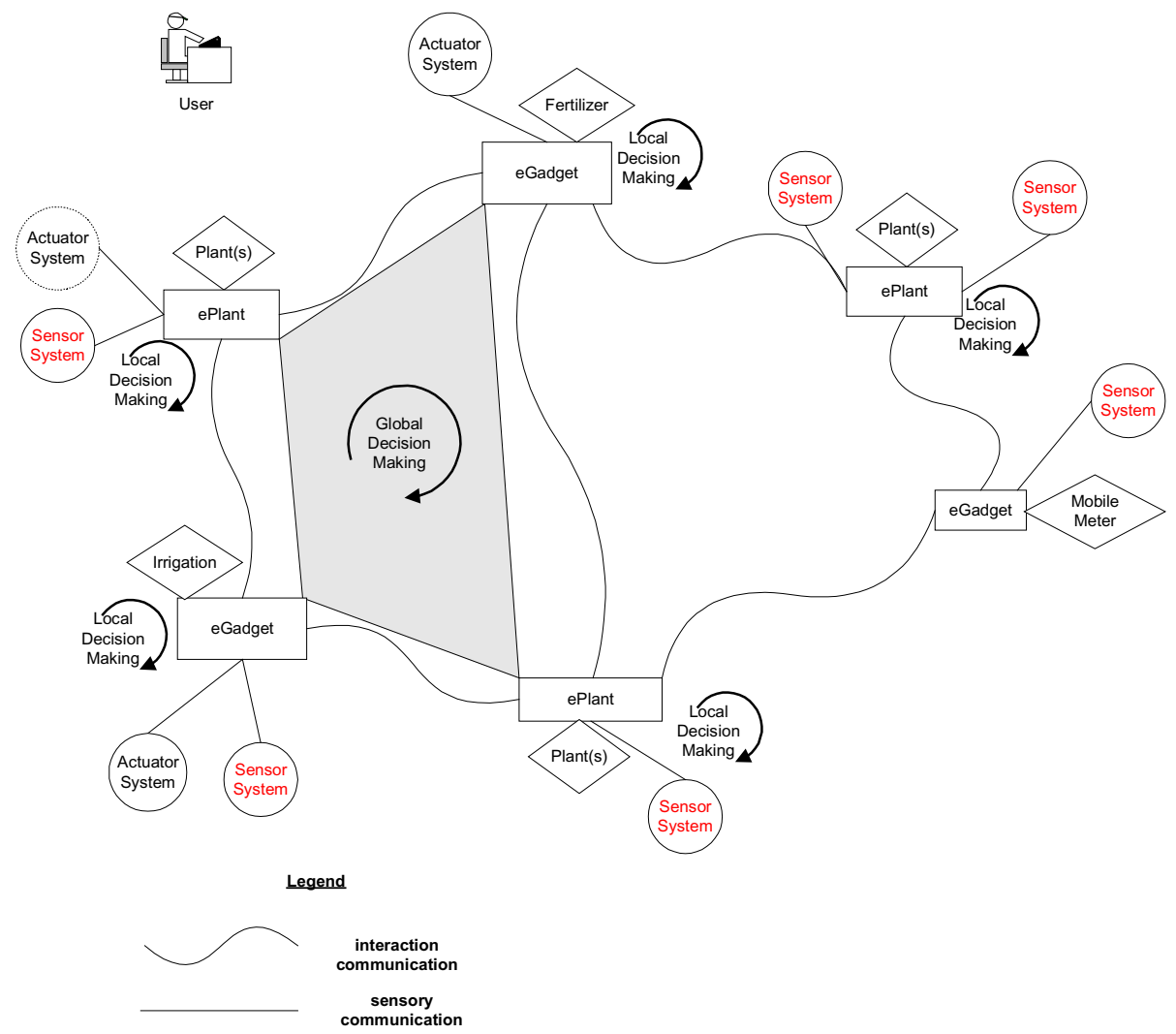

Fig. 2. An example of a bioGadgetworld, the functional operation of which defines a distributed system.

eGadgets/Actuators: eGadgets are GAS-enabled artefacts that may represent expressive devices (speakers, displays, mobile phones etc.), resource-providing devices (e.g. lamps, irrigation/fertilization/shading system) or any other everyday object. An actuator is part of the physical interface of a tangible object and actuator systems are mainly related to eGadgets nodes. The actuator systems will allow the plant to influence the environment that it resides in.

Sensor Systems: Sensor systems range from COTS devices to future microsensor networks, involving off-plant (non-contact) sensors. Individual sensor devices may be shared among a number of ePlants (e.g., due to cost constraints) so that the context needs to be determined. 


\section{Decision-Making}

The interaction of eGadgets and ePlants entails the triggering of local decisionmaking and global decision-making procedures. Upon determining the local state of a plant a decision may be required for the action to be followed. In the case of an eGadget (e.g., a lamp or a valve) the local decision-making (or resource management) mechanism resolves conflicts when multiple ePlants request a common resource (e.g., light or water). Distributed mechanisms can also be considered to alleviate similar situations, when ePlants and related eGadgets are coordinated for detecting/maintaining a global state/objective in the context of a group of distributed nodes (for example, is the growth of all plants in a domestic setting equally serviced? Or, is the whole field suffering a disease or a specific portion of it?)

The global state regarding a group of nodes will be a reasoning function based on the local states that are being monitored by the system. The nodes of the system have to communicate in order to exchange data. If we consider a group of nodes, a node is elected as 'coordinator' and gathers all the data that is needed in order to assess the global state. The algorithm is fully distributed so that in the case that the coordinator "goes off", another node will be elected as the coordinator node. The global state may not be as accurate as before but still it will be evaluated. When a global state is perceived then a global decision-making can be made/suggested.

\section{Sensory / Interaction Communication}

The communication in the distributed system is divided into two levels: sensory communication, which refers to the communication between a node and its sensor systems and the interaction communication, which refers to the communication between the nodes of the system.

We aim to separate the low-level sensory communication part from the interaction part. The former contains the sensors that transform chemical signals to digital and vice-versa and defines the lower layer of the distributed system. The latter one is used to conduct artefact / plant interaction which defines the higher layer of the distributed system. In that way, we separate the interaction services from the context of application (as defined by the sensor network communication).

\section{System Architecture}

A mixed society of communicating plants and artefacts forms a distributed system whose nodes are permeated by the illustrated architecture (Fig. 3). We have designed a layered modular architecture as to achieve a number of objectives, which would make the system more flexible and extensible.

At each layer, we have well-defined protocols that provide access to useful services: sensor data access, local state assessment, resource discovery, and so forth. At each layer, APIs may also be defined whose implementation exchange protocol messages with the appropriate service(s) to perform desired actions. 
At the heart of the architecture lie the middleware software layer (ePlantOS for ePlants / eGadgetOS for artefacts) that supports the deployment of mixed society applications by managing logical communication channels, called synapses, between the nodes of the distributed system. Both ePlantOS and eGadgetOS are sharing a common interaction module, understand basic concepts, and communicate by using common protocols and message structures. The functionality and services of the middleware is extended to fulfill the new requirements determined by the foreseen mixed societies and thus provides an interface with plant sensors and actuators, maintains an enhanced, plant-specific ontology and supports resource management and decisionmaking.

The I/O unit and connectivity layers administer the communication intricacies (e.g., commercial of the shelf sensor device communication protocols, routing protocols, etc.) in terms of the sensory and interaction communication views respectively of the system component.

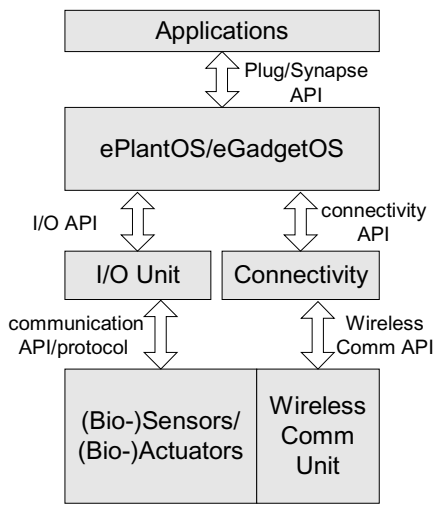

Fig. 3. System node architecture

A modular system design allows the replacement of a module without affecting the functionality of the rest provided that the APIs between them remain consistent. This principle holds for the different layers of the architecture as well as within each layer. The modular design of ePlantOS, for example, allows the integration of up-to-date algorithms and protocols in the form of plug-in modules.

We have decoupled the low-level sensory communication that gathers raw data from sensors from the application business logic, which we may consider, that is captured as a hierarchy of rules. In the same way we have separated the wireless communication networking issues from the application communication requirements. In other words our applications do not depend on a specific sensor or a protocol that this sensor uses to transfer data values. In that way, new sensor devices that emerge for a selected plant parameter can be integrated to the system without disturbing the other modules. In the same manner a different wireless communication protocol can be used without affecting the application business logic. Thus, we achieve technology-independence and adaptability.

In our approach, an application is realised through the cooperation of nodes of the distributed system in the form of established logical communication links between 
services and capabilities offered by the artefacts and the states and behaviours inferred from the plants (in each case services/states are provided through access points called plugs). The plug/synapse model [7] provides a conceptual abstraction that allows the user to describe mixed society ubiquitous applications. To achieve collective desired functionality, one forms synapses by associating compatible plugs, thus composing applications using eGadgets and ePlants as components. The use of highlevel abstractions, for expressing such associations, allows the flexible configuration and reconfiguration of mixed society applications with the use of appropriate editing tools.

In Fig. 4 we depict a simple mixed society of an ePlant associated with an eGadget (eLamp), so that when the chlorophyll fluorescence signal of the plant is below a certain level, implying a photo-oxidative stress situation, the light of the lamp must be turned on to a specific level of luminosity, until the chlorophyll fluorescence level rises up to a normal level again. A synapse has been formed between the Photoxidative Stress plug of the ePlant and the Light Intensity plug of the eLamp. The interaction module that implements the plug/synapse model is compatible between the two components and thus their interaction is feasible.

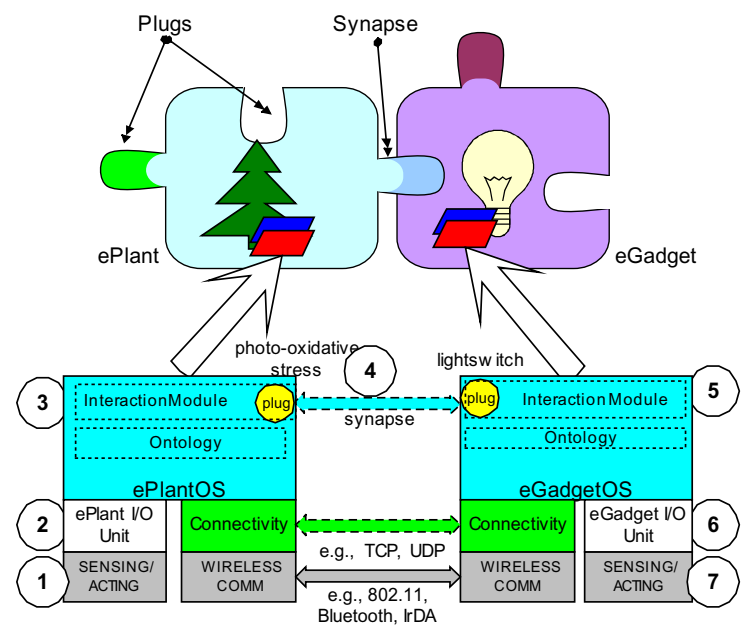

Fig. 4. An example of ePlant/eGadget interaction

In step 1, the biosensor/bioactuator network transforms selected plant (chlorophyll fluorescence) or other environmental (ambient light intensity) signals into digital signals. In step 2, ePlant's I/O Unit reads the digital signals (sensory communication), which will then be interpreted to a high-level unit of information, for instance, to an aggregated composite signal, and this information is transferred to the middleware (ePlantOS). In step 3 the context received by the middleware is applied to the rules encoded in the ontology so that a state of the plant is determined. Then the decision for an action may come in the form of a command or a request for a service. The ontology of the ePlantOS may specify, for example, the luminosity of the requested light, based on the plant species at hand. In step 4, the middleware passes the infor- 
mation to the connected eGadget through the established logical channel (synapse). The connectivity and wireless communication layers implement the lower layers of the network stack. Finally in steps 5-7 the middleware of the eGadget receives the information and acts upon the eGadget by using the eGadget $\mathrm{I} / \mathrm{O}$ unit that in turn activates an actuator through the sensor/actuator network.

\section{PLANTS Ontology}

The PLANTS system is designed so that it enables the semantically meaningful interaction between plants, artefacts and people via conceptualization of plants domain knowledge. The PLANTS ontology will represent the necessary knowledge in order to meet the PLANTS system's requirements and support its functionalities. We designed the PLANTS ontology keeping in mind two key issues described in the next paragraph; the first one is relevant to the way that we will exploit and use this ontology and the second one is relevant to the knowledge that this ontology should represent.

The PLANTS ontology will accommodate the semantic interoperability among heterogeneous ePlants and eGadgets by providing to them a common language. Thus the PLANTS ontology's first goal is to provide a formal representation of the domain under consideration, the bioGadgetWorlds (bioGWs). This representation demands the identification and the semantic description of the basic terms and concepts of the bioGWs as well as their interrelations. The basic concepts of the bioGWs are the following: eGadget (eGt), ePlant, Plug, Synapse, bioGW, Sensor, Actuator and Parameter. In the PLANTS ontology these concepts are represented as different classes, which have a number of properties. An eGt is a GAS enabled artefact. The properties of eGts are divided into two categories: the physical properties like shape, material, etc. and the digital properties like its plugs. As an eGt/ePlant exposes its services through plugs, a Plug represents the eGt/ePlant's capabilities. The connection between two Plugs is represented by a Synapse. A set of relations between these concepts represented in the PLANTS ontology are the following: an eGt/ePlant has Plugs, a Synapse is formed by exactly two Plugs, a bioGW contains at least two eGt/ePlants and a Synapse must exist between their plugs. In Fig. 5 we present the basic classes defined in PLANTS ontology and the associations between them.

One of the most important stages during the design of the PLANTS ontology was the definition of the ePlant. The digital-self of an ePlant doesn't differ to the one of an eGadget; thus the key issue is the representation of the physical-self of an ePlant, which is a specific plant or a group of plants. Each ePlant has a unique eEntityId, but the element that characterizes it is its name, which contains genus and species. Some other properties relevant to the plants that the ePlant contains are the following: the color and the type of its leaves, the color and shape of its flower, its developmental stages, its possible states, its symptoms and diseases and its stresses. 


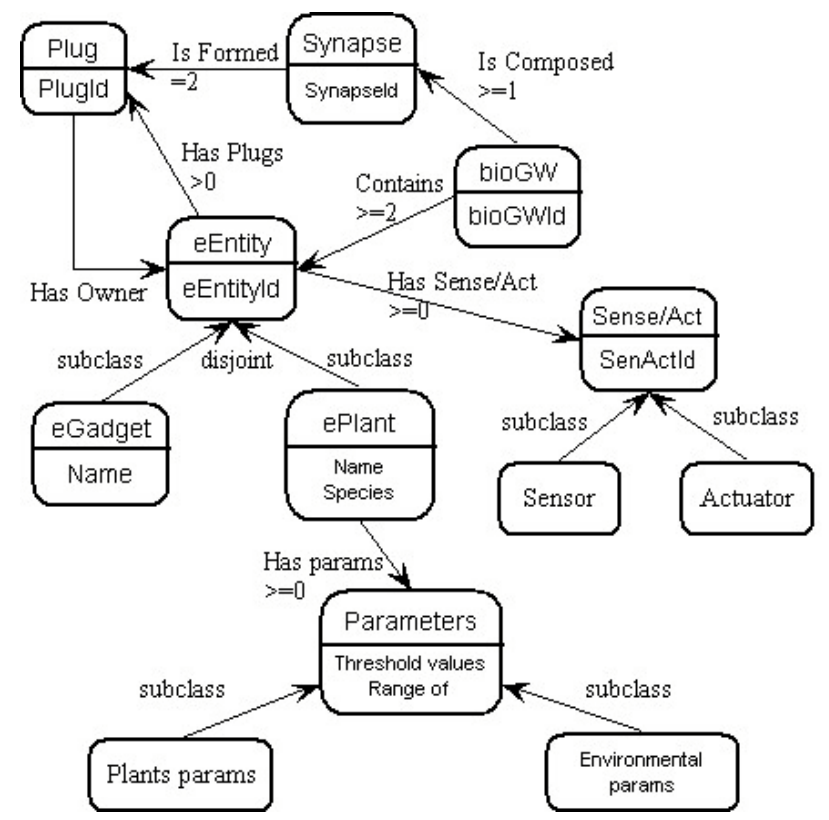

Fig. 5. Basic classes defined in PLANTS ontology and their associations

Another important role of the PLANTS ontology is to support both the local and global decision-making process. The decision-making process will be based on a set of rules in operational representation forms, which will be applied on existent knowledge and allow the use of the PLANTS ontology for reasoning providing inferential and validation mechanisms. The reasoning will be based on the definition of the PLANTS ontology, which may use simple description logic or user-defined reasoning using first-order logic. The PLANTS ontology should represent both the necessary knowledge and the appropriate rules. The knowledge that the PLANTS ontology needs in order to support the determination of the plant's state is emerged from the characterization of a plant and is strongly connected to the values of the plant parameters that are measured. So the plant parameters' definition and description are represented into the PLANTS ontology. Note that at an ePlant's definition there is an association between a plant parameter and at least a sensor. For the characterization of a plant's state and the decision-making process, the incorporation of environmental parameters into the PLANTS ontology will be helpful. Note that in order to have a complete view of a plant's characterization it is necessary to define the threshold values and/or the range of values for both plant and environmental parameters for different plant species and the states that these values imply.

PLANTS ontology refers to setting up a concept framework on how the knowledge about sensors, actuators and systems available on one hand and the biological studies about plant stressing and sensing mechanisms and consequent plant behaviour on the other hand can be formalized in order to make plants an active part of our ambient intelligence. The decision-making process based on the sensing of plants is also structured for the selected set of sensors and actuators and the correlated biological information allowing to interpret the plant behaviour. 


\section{$5 \quad$ Mixed Society Application Examples}

We aim to evaluate the development of the PLANTS system in a number of proof-ofconcept scenarios that range from domestic to outdoors UbiComp applications involving plants.

Discussions relating to the development of a scenario for the evaluation of the PLANTS system identified a number of categories relating to the interaction between plants, artefacts and people as follows:

\begin{tabular}{|l|l|}
\hline $\begin{array}{l}\text { Crop automa- } \\
\text { tion }\end{array}$ & $\begin{array}{l}\text { This type of scenario outlines the practical application of the } \\
\text { PLANTS technology for more sustainable agriculture and describes } \\
\text { plant signals being utilized to actuate the rate of individual agricul- } \\
\text { tural inputs (for example water and fertilizer). }\end{array}$ \\
\hline $\begin{array}{l}\text { Plant- } \\
\text { environmental } \\
\text { monitoring }\end{array}$ & $\begin{array}{l}\text { The status of a population of plants, either a monoculture or a mixed } \\
\text { population, could be envisaged to provide feedback to people on the } \\
\text { environmental conditions of a particular area. }\end{array}$ \\
\hline $\begin{array}{l}\text { Plant expres- } \\
\text { sion to man }\end{array}$ & $\begin{array}{l}\text { The devices around plants enable the communication of plant status, } \\
\text { in an abstract (i.e. changes in light, or the level of sound) or a more } \\
\text { explicit form (more detailed readings of the plant physiological } \\
\text { requirements). }\end{array}$ \\
\hline $\begin{array}{l}\text { Peer plant and } \\
\text { man }\end{array}$ & $\begin{array}{l}\text { This scenario category includes ideas that humanize plants. One } \\
\text { person is associated with one plant through a communication link, } \\
\text { whereby the plant status affects the person's environment (at home or } \\
\text { by some means of wearability). }\end{array}$ \\
\hline
\end{tabular}

Our collaborating work is towards creating two prototypes; one related to crop management and one related to an in-door scenario. The former will display how technological advance can promote and increase sustainability in agriculture and horticulture through the controlled monitoring of the plant (rather than of the physical environment only), to eliminate for example blanket pesticide treatments, up to excess water usage. The latter, involves the combined presence of plants and artefacts into an environment inhabited by humans (e.g. home, office, or public recreation spaces, etc) by exploiting plant signaling properties. A simple scenario for example is that when a plant feels "sick" or "hungry" it can notify the people at home/work or in a city park, trough spoken messages to their being taken care of. At home settings the plant itself can take actions for its well-being e.g., turning on/off the light or opening the window under certain conditions.

\section{Related Work}

The practical issues of building a UbiComp application, called PlantCare, that takes care of houseplants using a sensor network and a mobile robot are investigated in [10]. The emphasis is given on discussing technical challenges encountered during the deployment of the application. Our approach in contrast emphasizes the development of an architecture that views plants and associated computation as an integral part and allows the interaction of plants and artefacts in the form of synergistic and scaleable mixed societies. An ontology-based conceptual model is defined for composing Ubi- 
Comp applications which ensures a balanced behavior both to ambient nature applications where interactions through high level concepts and user empowerment is the focus, and agricultural nature applications where the integration of a large number of plant and environmental sensors and the complexity of the communication and the decision-making processes are the focal points.

LaughingLily presented in [11] is an artificial flower that is used as an ambient display. We support the same concept of using plants as a beautiful "encalming" interface or as a metaphor to convey information in a natural and unobtrusive manner. In the same time, the biological aspects of plants (e.g., photosynthetic analysis) can be equally brought forward for educational purposes or for environmental awareness.

Attempts to use environmental sensor networks in order to improve crops are reported in $[8,9]$. The approach taken is specific to crop management application whereas our approach strives to cover the ambient nature of applications equally. The later reference uses a centralized architecture to gather data followed by an analysis phase so that a grower becomes able to examine crop conditions in trial-and-error regime. This is in contrast to our distributed management system that allows both local and distributed decision-making approaches.

In the biology, botany, organic computing and bioinformatics domains there are activities on building ontologies $[12,13,14]$ that partially addressing principles of PLANTS. These activities aim to develop and share structured controlled vocabularies for plant-specific knowledge domains like plant anatomy, temporal stages, genes and biological sequences. Central to our approach is the use of an ontology, which provides not only a conceptual description of the domain knowledge, but furthermore the use of rules and constraints (axioms) in operational representation forms allow the use of the ontology for reasoning providing inferential and validation mechanisms. The reasoning is based on the definition of the ontology, which may use simple description logic or user-defined reasoning using first-order logic.

\section{Conclusions}

In this paper we discussed research work that enables the development of mixed societies of communicating plants and artefacts. The development of PLANTS ontology is a central point of our research. By means of developing a basic plant ontology, plants may become an active part of our ambient intelligence and they could become an information source and active members of a communication process with impact to plant crop management but also to domestics and other places where plants and humans have interfacing possibilities. Plants could provide additional sensing functionality to be used by humans for example as environmental quality markers. Eventually, it will enable totally new potential to decide about food plants quality during growth but also during food plant logistics for example from transportation up to the super market. Our immediate plans include the development of a suitable interface to "visualize" the ontology to the user. Demonstrating decision-making and plant monitoring processes based on such an interface to interested end-users we believe that would have a significant impact in terms of creating practical exploitation possibilities. 
Acknowledgement. This paper describes research carried within the PLANTS project (IST FET Open IST-2001-38900); more info can be found at http://www.edenproject/ PLANTS. The authors wish to thank their fellow researchers in the PLANTS consortium and the anonymous reviewers for their suggestions for improving this paper.

\section{References}

1. IST Advisory Group (ISTAG): Scenarios for Ambient Intelligence in 2010-full. February 2001, http://www.cordis.lu/ist/istag-reports.htm

2. Disappearing Computer initiative: http://www.disappearing-computer.net

3. e-Gadgets project website: http://www.extrovert-gadgets.net

4. Kameas A., et al.: An Architecture that Treats Everyday Objects as Communicating Tangible Components. In Proceedings of the 1st IEEE International Conference on Pervasive Computing and Communications (PerCom03), Fort Worth, USA, 2003.

5. PLANTS Consortium: PLANTS roadmap, PLANTS IST-2001-38900 Technical Report, April 2004.

6. Russell S. and Norvig P.: Artificial Intelligence: A Modern Approach. Prentice Hall, 2nd edition, 2003.

7. Mavrommati I., Kameas A. and Markopoulos P.: An Editing tool that manages device associations in an in-home environment. In Proceedings of the Second International Conference on Appliance Design (2AD), HP Labs, Bristol, UK, 11-13 May 2004, 104-111

8. Intel Research and Development: New Computing Frontiers - The Wireless Vineyard, http://www.intel.com/labs/features/rs01031.htm

9. PhyTech: Phytomonitoring TM, http://www.phytech.co.il/phyt.html

10. LaMarca A. et al.: PlantCare: An Investigation in Practical Ubiquitous Systems. 4th International Conference on Ubiquitous Computing (Springer-Verlag Lecture Notes in Computer Science Vol. 2498), Goteborg, Sweden, September 2002, 316-332.

11. Antifakos S., Schiele B.: LaughingLily: Using a Flower as a Real World Information Display. Poster at 5th International Conference on Ubiquitous Computing Seattle, Washington, USA, October 2003.

12. The Plant Ontology ${ }^{\mathrm{TM}}$ Consortium, http://www.plantontology.org

13. Gene Ontology Consortium, http://www.geneontology.org

14. Sequence Ontology, http://song.sourceforge.net 